\title{
Analysis of an Improved IMU-based Observer for Multirotor Helicopters
}

John Charles Macdonald

Brigham Young University - Provo, jandkmacdonald6@gmail.com

Robert C. Leishman

Brigham Young University - Provo, rleish@gmail.com

Randal W. Beard

Brigham Young University - Provo, beard@byu.edu

Timothy W. McLain

Brigham Young University - Provo, mclain@byu.edu

Follow this and additional works at: https://scholarsarchive.byu.edu/facpub

Part of the Electrical and Computer Engineering Commons, and the Mechanical Engineering

Commons

\section{Original Publication Citation}

Macdonald, J., Leishman, R., Beard, R. and McLain, T. Analysis of an Improved IMU-based Observer for Multirotor Helicopters, Journal of Intelligent and Robotic Systems, DOI 10.1007/ s10846-013-9835-5, May 2013.

\section{BYU ScholarsArchive Citation}

Macdonald, John Charles; Leishman, Robert C.; Beard, Randal W.; and McLain, Timothy W., "Analysis of an Improved IMU-based Observer for Multirotor Helicopters" (2013). Faculty Publications. 1517.

https://scholarsarchive.byu.edu/facpub/1517

This Peer-Reviewed Article is brought to you for free and open access by BYU ScholarsArchive. It has been accepted for inclusion in Faculty Publications by an authorized administrator of BYU ScholarsArchive. For more information, please contact ellen_amatangelo@byu.edu. 


\title{
Analysis of an Improved IMU-Based Observer for Multirotor Helicopters
}

\author{
John Macdonald • Robert Leishman • \\ Randal Beard . Timothy McLain
}

Received: date / Accepted: date

\begin{abstract}
Multirotor helicopters are increasingly popular platforms in the robotics community. Making them fully autonomous requires accurate state estimation. We review an improved dynamic model for multirotor helicopters and analyze the observability properties of an estimator based on this model. The model allows better use of IMU data to facilitate accurate state estimates even when updates from a sensor measuring position become less frequent and less accurate. We demonstrate that the position update rate can be cut in half versus typical approaches while maintaining the same accuracy. We also
\end{abstract}

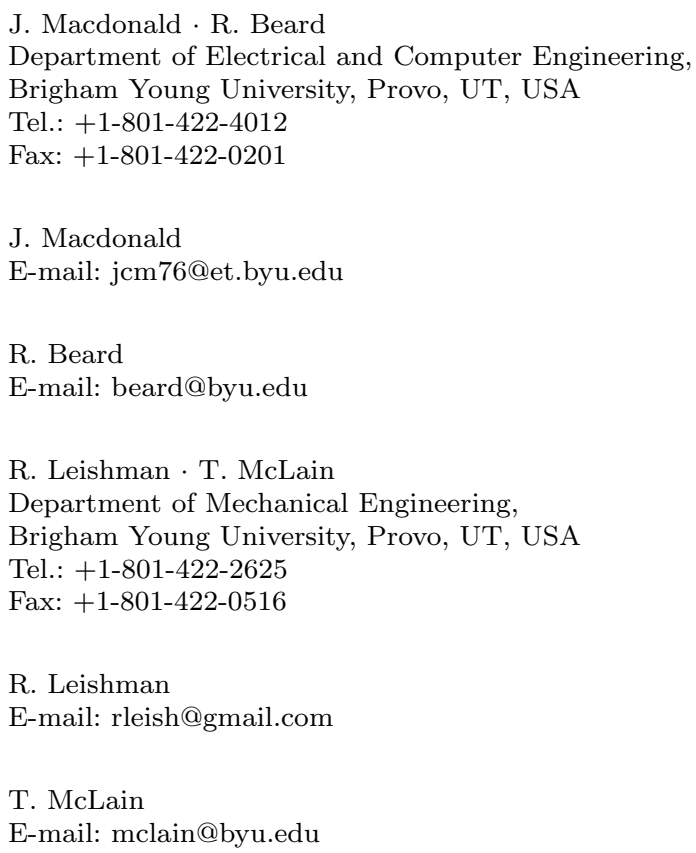


find that velocity estimates are at least twice as accurate no matter what the position update rate.

Keywords Multirotor Helicopter - Quadrotor · State Estimation · Observability

\section{Introduction}

Multirotor helicopters have been identified as potential "game changers in robotics" [8]. They have already been used to accomplish complex missions and are favored for their maneuverability and mechanical simplicity. Further development will open the door to a host of commercial and military applications such as search and rescue or disaster response in unstructured and adverse environments.

We believe estimating the state (position, orientation, and velocity) of a multirotor helicopter is the primary hurdle to enabling truly autonomous path planning and control. This task is relatively easy in an environment with known structure or when using GPS or other external reference systems. Several researchers use such assumptions and systems to simplify estimation, leading to sophisticated planning [2], [6], [17] and control [14], [15] for multirotor vehicles.

However, if the environment is unknown and a system like GPS is not available then state estimation becomes considerably more difficult. The vehicle must build up its own globally consistent map of the environment while using the same map to localize itself. In other words, the vehicle must perform simultaneous localization and mapping (SLAM), and SLAM algorithms tend to require significant computational resources. An introduction to SLAM is beyond the scope of this paper; we refer the reader to tutorial material on the topic (e.g. [20]). ${ }^{1}$

A few research groups have made noteworthy progress toward deploying fully autonomous multirotor helicopters capable of operating in unknown environments. The authors of [1] present their system with the comment that, "one of the major challenges ... is estimating the position and velocity." For these estimates they rely on accurate and frequent position measurements based on a SLAM map produced from laser scan data. They mention that they "require a much higher map resolution" because of their velocity estimation approach. Their laser scan matching algorithm uses the map to generate position updates at $40 \mathrm{~Hz}$; velocity estimates are only updated indirectly. Their SLAM algorithm requires an offboard computer for processing.

In [19] the authors present a four rotor helicopter (i.e. quadrotor) that also uses laser scan matching and a SLAM map. They state that, "the vehicle dynamics require pose estimates with update rates of $20 \mathrm{~Hz}$." Their velocity estimates are only corrected by their kinematic relationship to position.

\footnotetext{
1 Our approach and results are also applicable to a vehicle with access to GPS; our work is agnostic to the source of position information. However, the results showing a decrease in the dependence on position updates are especially relevant in the context of complete autonomy from any offboard system.
} 
Because all processing is performed onboard, several of the design decisions detailed in [19] are driven by the need to meet the system's computational limitations.

The authors of [13] rely on the increased information available from cameras at the expense of increased computation. They emphasize that "the estimated velocity is critical to damp the system." They briefly present a state estimation approach which uses a constant-velocity motion model to predict velocity states between vision measurements. The vision algorithm measures position within a known map. They note that the simplistic prediction model raises the minimum update rate the filter requires from the vision processing.

We can draw several points of consensus from the preceding work. Each agrees that state estimation, especially velocity estimation, must be fast and accurate to enable autonomous control. They also all approach the state estimation problem by relying on frequent position updates. Their algorithms providing consistent position information tax the vehicle's limited onboard computers, leading to a dependence on offboard systems [1], stringent design constraints [19], or the assumption of a known map [13].

Recent work with multi-rotor helicopters further emphasizes the importance of accurate velocity estimates in the plane perpendicular to gravity. The authors of [18] present system identification and estimation results for a quadrotor. They note that, "Translational damping is more problematic since there is no translational velocity sensor. One effective approach is differentiating high-rate position information.... However when the position measurements occur at a low rate, for example from a light-weight onboard laser scanner or camera, the resulting lag in the velocity estimate is highly destabilising."

Our contribution in this paper is to present and analyze an observer that approaches the state estimation problem from a different perspective: improving velocity and attitude estimates by more accurately using IMU measurements. The improvement comes through a better dynamic model that correctly explains the physics related to the velocity. As an immediate consequence of the model, we confirm that the accelerometers directly measure the translational velocity. We also show how improved information on velocity relaxes demands on the computationally expensive algorithms that give position estimates. To our knowledge, nothing in the literature quantifies the improvement in state estimation afforded by using accelerometer measurements to directly update velocity estimates. In our results we compare our estimates to truth as well as to estimates from more traditional approaches to make a quantitative comparison.

The rest of the paper is organized as follows. In Section 2 we provide an overview of the model used in our observer. We then present a nonlinear observability analysis in Section 3. We present our results in Section 4, and we make concluding remarks in Section 5. 


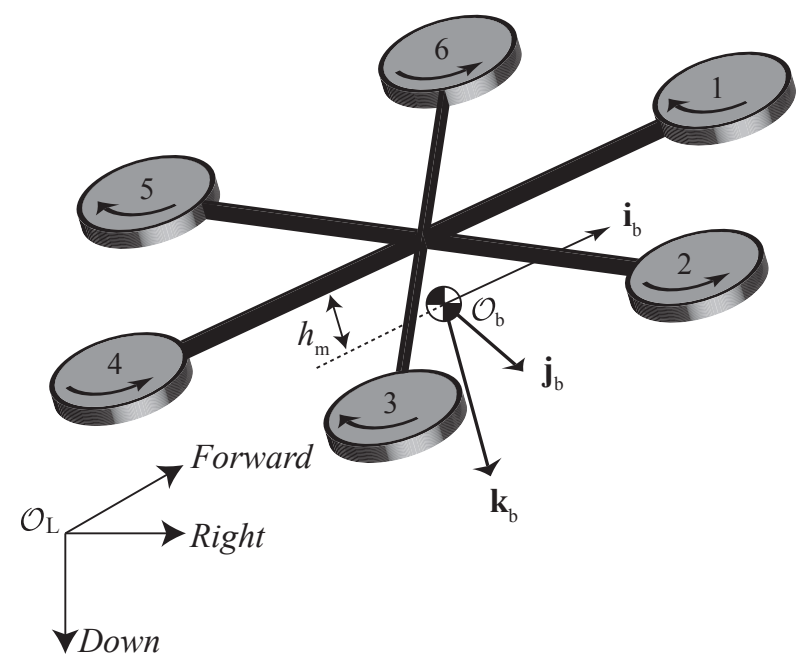

Fig. 1 Schematic of a six-rotor helicopter, or hexacopter. The hexacopter's center of mass is a distance $h_{\mathrm{m}}$ directly below the vehicle's geometric center, coincident with the body-fixed reference frame origin, $\mathcal{O}_{\mathrm{b}}$. We define the body-fixed reference frame $\mathbf{i}_{\mathrm{b}}$ axis to be in the plane normal to $h_{\mathrm{m}}$ and in the direction of motor one. The body $\mathbf{j}_{\mathrm{b}}$ axis is in the same plane pointing to the vehicle's right, and the $\mathbf{k}_{\mathrm{b}}$ axis completes a right-hand coordinate system. Navigation is with respect to a fixed local reference frame with origin $\mathcal{O}_{\mathrm{L}}$. The local reference frame down axis is parallel to gravity; the vehicle's horizontal position is given by a forward and right displacement from $\mathcal{O}_{\mathrm{L}}$.

\section{Observer Description}

We seek to estimate the position, orientation, and linear velocity of a commercially available six rotor helicopter (i.e. hexacopter) along with several IMU sensor biases. Figure 1 shows a schematic representation of the vehicle and important notation. The approach we present can be readily adapted to other multirotor helicopters.

\subsection{Model Definition}

We define the state vector to be

$$
\mathbf{x} \triangleq\left[\begin{array}{lllllllllllllll}
f_{\mathrm{L}} & r_{\mathrm{L}} & d_{\mathrm{L}} & \phi & \theta & \psi & u & v & w & \beta_{\mathrm{i}_{\mathrm{b}}} & \beta_{\mathrm{j}_{\mathrm{b}}} & \beta_{\mathrm{k}_{\mathrm{b}}} & \alpha_{\mathrm{i}_{\mathrm{b}}} & \alpha_{\mathrm{j}_{\mathrm{b}}}
\end{array}\right]^{\top},
$$

where $f_{\mathrm{L}}, r_{\mathrm{L}}$, and $d_{\mathrm{L}}$ represent the vehicle's forward, right, and down displacement from a local reference frame; $\phi, \theta$, and $\psi$ are the roll, pitch, and yaw angles relating the local reference frame to the body-fixed reference frame; $u$, $v$, and $w$ are the hexacopter's linear velocity resolved in the body $\mathbf{i}_{\mathrm{b}}, \mathbf{j}_{\mathrm{b}}$, and $\mathbf{k}_{\mathrm{b}}$ axes; $\beta_{\mathrm{i}_{\mathrm{b}}}, \beta_{\mathrm{j}_{\mathrm{b}}}$, and $\beta_{\mathrm{k}_{\mathrm{b}}}$ are the biases in gyroscopes respectively measuring angular velocity about the body $\mathbf{i}_{\mathrm{b}}, \mathbf{j}_{\mathrm{b}}$, and $\mathbf{k}_{\mathrm{b}}$ axes; and $\alpha_{\mathrm{i}_{\mathrm{b}}}$ and $\alpha_{\mathrm{j}_{\mathrm{b}}}$ are biases 
in the accelerometers respectively aligned with the $\mathbf{i}_{\mathrm{b}}$ and $\mathbf{j}_{\mathrm{b}}$ axes. The rotation matrix from the body-fixed to the local reference frame is

$$
\begin{aligned}
\mathbf{R}_{\mathrm{b}}^{\mathrm{L}} & \triangleq\left[\begin{array}{ccc}
r_{11} & r_{12} & r_{13} \\
r_{21} & r_{22} & r_{23} \\
r_{31} & r_{32} & r_{33}
\end{array}\right] \\
& \triangleq\left[\begin{array}{ccc}
\mathrm{c}_{\theta} \mathrm{c}_{\psi} & \mathrm{s}_{\phi} \mathrm{s}_{\theta} \mathrm{c}_{\psi}-\mathrm{c}_{\phi} \mathrm{s}_{\psi} & \mathrm{c}_{\phi} \mathrm{s}_{\theta} \mathrm{c}_{\psi}+\mathrm{s}_{\phi} \mathrm{s}_{\psi} \\
\mathrm{c}_{\theta} \mathrm{s}_{\psi} & \mathrm{s}_{\phi} \mathrm{s}_{\theta} \mathrm{s}_{\psi}+\mathrm{c}_{\phi} \mathrm{c}_{\psi} & \mathrm{c}_{\phi} \mathrm{s}_{\theta} \mathrm{s}_{\psi}-\mathrm{s}_{\phi} \mathrm{c}_{\psi} \\
-\mathrm{s}_{\theta} & \mathrm{s}_{\phi} \mathrm{c}_{\theta} & \mathrm{c}_{\phi} \mathrm{c}_{\theta}
\end{array}\right],
\end{aligned}
$$

where $\mathrm{c}_{\theta} \triangleq \cos (\theta), \mathrm{s}_{\theta} \triangleq \sin (\theta)$, etc.

The state propagation and measurement equations

$$
\begin{aligned}
\dot{\mathbf{x}} & =\mathbf{f}(\mathbf{x}, \mathbf{u}), \\
y_{n} & =h_{n}(\mathbf{x}),
\end{aligned}
$$

characterize the model and are further defined below. The vector $\mathbf{u}$ represents the inputs to the model. We use gyroscope measurements to predict the evolution of state estimates. Therefore,

$$
\mathbf{u} \triangleq\left[\begin{array}{c}
\gamma_{\mathrm{i}_{\mathrm{b}}} \\
\gamma_{\mathrm{j}_{\mathrm{b}}} \\
\gamma_{\mathrm{k}_{\mathrm{b}}}
\end{array}\right]=\left[\begin{array}{c}
p+\beta_{\mathrm{i}_{\mathrm{b}}} \\
q+\beta_{\mathrm{j}_{\mathrm{b}}} \\
r+\beta_{\mathrm{k}_{\mathrm{b}}}
\end{array}\right],
$$

where $\gamma_{\mathrm{i}_{\mathrm{b}}}, \gamma_{\mathrm{j}_{\mathrm{b}}}$, and $\gamma_{\mathrm{k}_{\mathrm{b}}}$ are the gyroscope measurements about the subscripted axes, and $p, q$, and $r$ are the actual rotation rates. We model the time evolution of each bias state as a random walk and define the rest of Eq. (3) as

$$
\begin{aligned}
& {\left[\begin{array}{c}
\dot{f}_{\mathrm{L}} \\
\dot{r}_{\mathrm{L}} \\
\dot{d}_{\mathrm{L}}
\end{array}\right]=\mathbf{R}_{\mathrm{b}}^{\mathrm{L}}\left[\begin{array}{c}
u \\
v \\
w
\end{array}\right],} \\
& {\left[\begin{array}{c}
\dot{\phi} \\
\dot{\theta} \\
\dot{\psi}
\end{array}\right]=\left[\begin{array}{ccc}
1 & \sin \phi \tan \theta & \cos \phi \tan \theta \\
0 & \cos \phi & -\sin \phi \\
0 & \frac{\sin \phi}{\cos \theta} & \frac{\cos \phi}{\cos \theta}
\end{array}\right]\left[\begin{array}{c}
p \\
q \\
r
\end{array}\right],} \\
& {\left[\begin{array}{c}
\dot{u} \\
\dot{v} \\
\dot{w}
\end{array}\right]=\left[\begin{array}{ccc}
-g \sin \theta+(v r-w q)-\frac{F_{\mathrm{di}}}{m} \\
g \sin \phi \cos \theta+(w p-u r)-\frac{F_{\mathrm{dj}}}{m} \\
g \cos \phi \cos \theta+(u q-v p)-\frac{T}{m}
\end{array}\right],}
\end{aligned}
$$

where $g$ is the acceleration due to gravity, $m$ is the vehicle's mass, and $T$ is the cumulative thrust of the motors.

Equation (8) is particularly important to this paper. The forces on the right-hand side can be attributed to gravity, the Coriolis force, thrust, and drag. The variables $F_{\mathrm{di}}$ and $F_{\mathrm{dj}}$ are the drag forces, and they represent the small but important deviation from the majority of multirotor helicopter models in the literature. We discuss the cause and importance of these drag forces below. 
2.2 Drag Force and Accelerometer Measurements

Drag is often ignored when modeling multirotor helicopters. Many papers acknowledge that some drag force must act on the vehicle's body, but this is reasonably dismissed. The drag on the body is proportional to its cross-sectional area as well as the square of its linear velocity. The area and velocity are both typically small for a multirotor helicopter. In particular, velocity is limited to maintain the quality of onboard sensor measurements such as images or laser scans. Ignoring drag leads most researchers to assume a dynamic model that only accounts for gravity, Coriolis, and thrust forces (e.g. [4]).

Other researchers include in the dynamic model a drag force that is directly proportional to the multirotor helicopter's linear velocity. For example, [10], [22], and [11] identify such a term. However, these authors' emphasis on control algorithms avoids any discussion of the physics that generate the drag or its effect on accelerometers or estimation. Bouabdallah [3] touches on the nature of such a drag force, but he uses it only for modeling and simulation. Like the preceding authors, Bouabdallah's emphasis on control algorithms leads to an implementation that ignores the drag term.

The authors of [1] model a drag force proportional to linear velocity in their state estimation approach. They include the term as an expediency based on the authors' observation that something must prevent the quadrotor from accelerating indefinitely. They offer no physical explanation for the effect and instead rely on a motion capture system and system identification techniques to estimate the proportionality constant. The relationship between drag and the accelerometer measurements is left unclear in their very brief discussion of IMU measurements.

The drag terms $F_{\mathrm{di}}$ and $F_{\mathrm{dj}}$ from Equation (8) are in fact proportional to linear velocity, and they are due to rotor drag. It is reasonable to ignore them when designing a controller because they are relatively small in magnitude when flying at practical speeds. However, accounting for these drag forces is significant in state estimation.

The authors of [12] were the first to describe $F_{\mathrm{di}}$ and $F_{\mathrm{dj}}$ in detail. In their paper they analytically develop the entire dynamic model of a quadrotor at great length, beginning with fundamental blade element theory. However, their experimental validation of the model is limited. They conclude with a discussion of two controllers based on their drag-force enhanced model. The resulting hardware implementations are only assessed qualitatively by stating that the systems were "much easier to fly than with the usual scheme."

The important principle we share with [12] is to identify $F_{\mathrm{di}}$ and $F_{\mathrm{dj}}$ with the accelerometer measurements. The $\mathbf{i}_{\mathrm{b}}$ and $\mathbf{j}_{\mathrm{b}}$ axis accelerometers measure the specific forces (i.e. total force minus the effect of gravity) associated with $\dot{u}$ and $\dot{v}$. The specific forces modeled in (8) include Coriolis terms and $F_{\mathrm{di}}$ and $F_{\mathrm{dj}}$. The Coriolis terms are relatively small for autonomous flight, again because the vehicle cannot translate or rotate quickly without rendering exteroceptive sensor data useless. Therefore we can model the $\mathbf{i}_{\mathrm{b}}$ and $\mathbf{j}_{\mathrm{b}}$ axis accelerometer 
measurements respectively as

$$
\begin{aligned}
& h_{1}(\mathbf{x}) \triangleq-\frac{F_{\mathrm{di}}}{m}=-\frac{\mu}{m} u, \\
& h_{2}(\mathbf{x}) \triangleq-\frac{F_{\mathrm{dj}}}{m}=-\frac{\mu}{m} v,
\end{aligned}
$$

where $\mu$ is an aerodynamic term we discuss further below. The important point here is that these accelerometers offer a direct, scaled measurement of the corresponding velocity components.

The aerodynamic term $\mu$ can depend on several factors, but for nominal autonomous flight conditions $\mu$ can be treated as a constant. The correct value for $\mu$ can be estimated directly given knowledge of the velocity components $u$ and $v$, such as from a motion capture system. Measured values of $u$ and $v$ can be used to find a least-squares fit of (9) and (10) to the actual accelerometer measurements. Using this approach to estimate $\mu$, we demonstrate in Figure 2 the agreement between actual accelerometer measurements and the measurement models defined in (9) and (10).
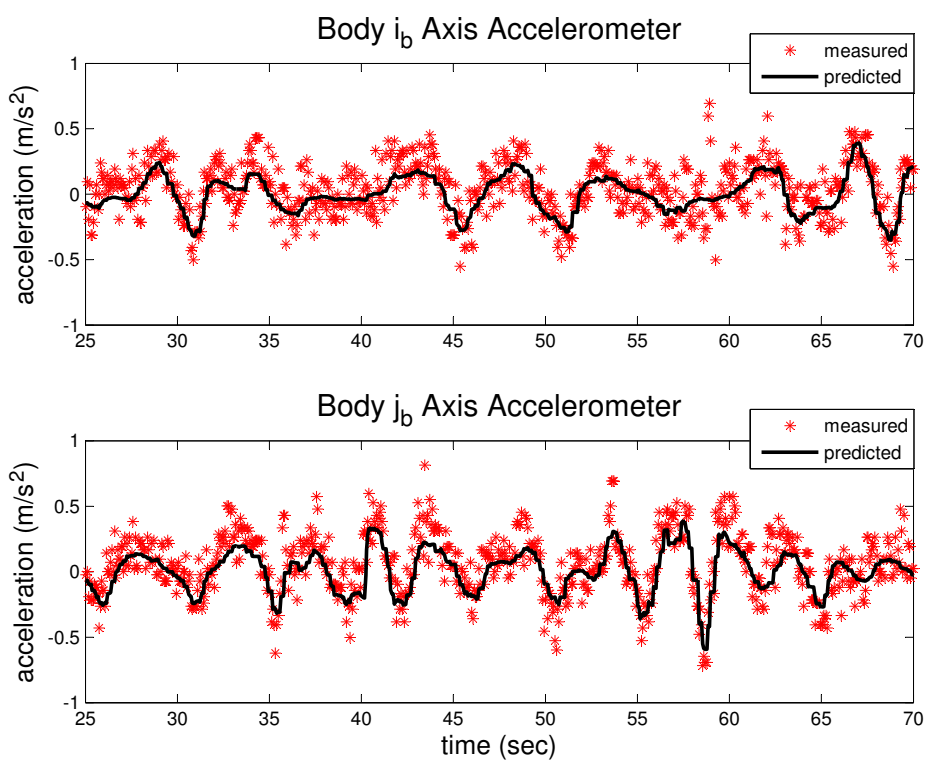

Fig. 2 Actual accelerometer measurements for a nominal indoor flight plotted against those predicted by (9) and (10).

\subsection{Observer Definition}

We now define the observer implemented in this paper to estimate the state defined in Equation (1). We implement the observer as a Continuous-Discrete 
Extended Kalman Filter (CD-EKF). In a CD-EKF the state estimates are numerically propagated forward in time as continuous variables. When a measurement becomes available it is applied as a discrete time input to the filter.

Specifically, let $T_{o}$ be the period at which the CD-EKF outputs its state estimate, $\hat{\mathbf{x}}$. The period $T_{o}$ is chosen to be much less than the interval between measurement updates. For each output from the CD-EKF we have

$$
\begin{aligned}
\hat{\mathbf{x}} & =\hat{\mathbf{x}}+T_{o} \mathbf{f}(\hat{\mathbf{x}}, \mathbf{u}), \\
\mathbf{A} & =\frac{\partial \mathbf{f}}{\partial \mathbf{x}}(\hat{\mathbf{x}}, \mathbf{u}), \\
\mathbf{B} & =\frac{\partial \mathbf{f}}{\partial \mathbf{u}}(\hat{\mathbf{x}}, \mathbf{u}), \\
\mathbf{P} & =\mathbf{P}+T_{o}\left(\mathbf{A P}+\mathbf{P A}^{\top}+\mathbf{B G B}^{\top}+\mathbf{Q}\right),
\end{aligned}
$$

where $\mathbf{P}$ is the filter estimated covariance, $\mathbf{G}$ is the covariance of the three gyroscopes ${ }^{2}$ used as inputs to the observer, and $\mathbf{Q}$ is a diagonal, hand tuned matrix primarily used to model the random walk propagation of the bias states.

When a measurement from the $n^{\text {th }}$ sensor is available the CD-EKF updates its state estimates with that sensor information according to

$$
\begin{aligned}
\mathbf{C}_{n} & =\frac{\partial h_{n}}{\partial \mathbf{x}}(\hat{\mathbf{x}}, \mathbf{u}), \\
\mathbf{L}_{n} & =\mathbf{P C}_{n}^{\top}\left(R_{n}+\mathbf{C}_{n} \mathbf{P C}_{n}^{\top}\right)^{-1}, \\
\mathbf{P} & =\mathbf{P}-\mathbf{L}_{n} \mathbf{C}_{n} \mathbf{P}, \\
\hat{\mathbf{x}} & =\hat{\mathbf{x}}+\mathbf{L}_{n}\left(y_{n}-h_{n}(\hat{\mathbf{x}})\right),
\end{aligned}
$$

where $R_{n}$ is the uncertainty associated with the $n^{\text {th }}$ sensor, and $y_{n}$ is the actual measurement from the $n^{\text {th }}$ sensor. For the remainder of this paper we assume that the CD-EKF has access to the two accelerometer measurements modeled by Equations (9) and (10) as well as position and heading measurements from an appropriate SLAM algorithm.

In Section 4 we show that using the above model for accelerometer measurements significantly improves estimates of the velocities $u$ and $v$. Roll and pitch estimates also especially benefit because those states cause the hexacopter's lateral acceleration. But first we analytically demonstrate the reason for these improvements by examining the observability of (3) and (4).

\section{Observability Analysis}

Observability is a necessary condition for an filter to converge. Examining a filter's observability properties can also shed light on why a filter behaves

\footnotetext{
2 The covariance matrix $\mathbf{G}$ is assumed to be a constant diagonal matrix. Its nonzero entries are estimated a priori by measuring the noise characteristics of the individual gyroscopes. Mapping this noise through the Jacobian B makes the filter easy to tune and more accurate. See [9] for more detail.
} 
in certain ways. The following presentation is self-contained, but for more background on nonlinear observability we refer the reader to the textbook by Vidyasagar [21].

Let $X$ denote an open subset of $\mathbb{R}^{14}$. Let $S(X)$ and $V(X)$ respectively designate the set of all scalar valued smooth functions and the set of all vector fields (i.e. column vectors of smooth functions) on $X$. For the observability analysis it is convenient to rewrite (3) as

$$
\dot{\mathbf{x}}=\mathbf{f}(\mathbf{x})+\sum_{j=1}^{3} u_{j} \mathbf{g}_{j}(\mathbf{x})
$$

with $\mathbf{f}, \mathbf{g}_{j} \in V(X)$. In addition to the accelerometer measurements given in (9) and (10), we assume an appropriate algorithm provides the measurements

$$
\begin{aligned}
& h_{3} \triangleq f_{\mathrm{L}}, \\
& h_{4} \triangleq r_{\mathrm{L}}, \\
& h_{5} \triangleq d_{\mathrm{L}}, \\
& h_{6} \triangleq \psi,
\end{aligned}
$$

where $h_{n} \in S(X)$.

To facilitate the discussion, recall that the Lie derivative of a function $h_{n} \in S(X)$ with respect to any vector field $\mathbf{q} \in V(X)$ is defined by the mapping

$$
\mathbf{x} \mapsto \mathbf{d} h_{n}(\mathbf{x}) \cdot \mathbf{q}(\mathbf{x}): X \rightarrow \mathbb{R}
$$

where $\mathbf{d} h_{n}$ is the gradient of $h_{n}$ with respect to $\mathbf{x}$. This Lie derivative is given the notation $L_{\mathbf{q}} h_{n}$. Note that the result of the Lie derivative is also a scalar valued function of $\mathbf{x}$.

For the nonlinear system (3) - (4) to be locally observable at a given point $\overline{\mathbf{x}} \in X$, it is sufficient to show that there exist 14 linearly independent row vectors in the set

$$
\left\{\left.\mathbf{d} h_{n}\right|_{\overline{\mathbf{x}}}\right\} \cup\left\{\left.\mathbf{d} L_{\mathbf{z}_{s}} L_{\mathbf{z}_{s-1}} \cdots L_{\mathbf{z}_{1}} h_{n}\right|_{\overline{\mathbf{x}}}\right\}
$$

where $1 \leq n \leq 6, s \geq 1$, and $\mathbf{z}_{j} \in\left\{\mathbf{f}, \mathbf{g}_{1}, \mathbf{g}_{2}, \mathbf{g}_{3}\right\}$. In other words, if one can find enough linearly independent vectors in the gradient of the measurement equations or in the gradient of their Lie derivatives, the system is locally observable at that point.

It is easy to see that the six row vectors of the first type $\left(\left.\mathbf{d} h_{n}\right|_{\overline{\mathbf{x}}}\right)$ constitute a set of six linearly independent rows. We next examine the six additional vectors $\mathbf{d} L_{\mathbf{f}} h_{1: 6}$. We represent a vector of zeros of length $\mathrm{m}$ as $\mathbf{0}_{\mathrm{m}}$ to make the expressions more compact. We also use the element-wise definition of $\mathbf{R}_{\mathrm{b}}^{\mathrm{L}}$ from 
(2). This gives

$$
\begin{aligned}
& \mathbf{d} L_{\mathbf{f}} h_{1}=\left[\begin{array}{c}
\mathbf{0}_{3} \\
0 \\
\frac{g \mu}{m} \mathrm{c}_{\theta} \\
0 \\
\frac{\mu^{2}}{m^{2}} \\
\frac{\mu}{m} \beta_{\mathrm{k}_{\mathrm{b}}} \\
-\frac{\mu}{m} \beta_{\mathrm{j}_{\mathrm{b}}} \\
0 \\
-\frac{\mu}{m} w \\
\frac{\mu}{m} v \\
\mathbf{0}_{2}
\end{array}\right]^{\top}, \quad \mathbf{d} L_{\mathbf{f}} h_{2}=\left[\begin{array}{c}
\mathbf{0}_{3} \\
-\frac{g \mu}{m} \mathrm{c}_{\phi} \mathrm{c}_{\theta} \\
\frac{g \mu}{m} \mathbf{s}_{\phi} \mathbf{s}_{\theta} \\
0 \\
-\frac{\mu}{m} \beta_{\mathrm{k}_{\mathrm{b}}} \\
\frac{\mu^{2}}{m^{2}} \\
\frac{\mu}{m} \beta_{\mathrm{i}_{\mathrm{b}}} \\
\frac{\mu}{m} w \\
0 \\
-\frac{\mu}{m} u \\
\mathbf{0}_{2}
\end{array}\right] \\
& \mathbf{d} L_{\mathbf{f}} h_{3}=\left[\begin{array}{c}
\mathbf{0}_{3} \\
v \frac{\partial r_{12}}{\partial \phi}+w \frac{\partial r_{13}}{\partial \phi} \\
u \frac{\partial r_{11}}{\partial \theta}+v \frac{\partial r_{12}}{\partial \theta}+w \frac{\partial r_{13}}{\partial \theta} \\
u \frac{\partial r_{11}}{\partial \psi}+v \frac{\partial r_{12}}{\partial \psi}+w \frac{\partial r_{13}}{\partial \psi} \\
r_{11} \\
r_{12} \\
r_{13} \\
\mathbf{0}_{5}
\end{array}\right]^{\top} \quad \mathbf{d} L_{\mathbf{f}} h_{4}=\left[\begin{array}{c}
\mathbf{0}_{3} \\
v \frac{\partial r_{22}}{\partial \phi}+w \frac{\partial r_{23}}{\partial \phi} \\
u \frac{\partial r_{21}}{\partial \theta}+v \frac{\partial r_{22}}{\partial \theta}+w \frac{\partial r_{23}}{\partial \theta} \\
u \frac{\partial r_{21}}{\partial \psi}+v \frac{\partial r_{22}}{\partial \psi}+w \frac{\partial r_{23}}{\partial \psi} \\
r_{21} \\
r_{22} \\
r_{23} \\
\mathbf{0}_{5}
\end{array}\right]^{\top} \\
& \mathbf{d} L_{\mathbf{f}} h_{5}=\left[\begin{array}{c}
\mathbf{0}_{3} \\
v \frac{\partial r_{32}}{\partial \phi}+w \frac{\partial r_{33}}{\partial \phi} \\
u \frac{\partial r_{31}}{\partial \theta}+v \frac{\partial r_{32}}{\partial \theta}+w \frac{\partial r_{33}}{\partial \theta} \\
0 \\
r_{31} \\
r_{32} \\
r_{33} \\
\mathbf{0}_{5}
\end{array}\right]^{\top}, \quad \mathbf{d} L_{\mathbf{f}} h_{6}=\left[\begin{array}{c}
\mathbf{0}_{3} \\
-\frac{\mathrm{c}_{\phi}}{\mathrm{c}_{\theta}} \beta_{\mathrm{j}_{\mathrm{b}}}+\frac{\mathbf{s}_{\phi}}{\mathrm{c}_{\theta}} \beta_{\mathrm{k}_{\mathrm{b}}} \\
-\frac{\mathrm{s}_{\phi} \mathbf{s}_{\theta}}{\left(\mathrm{c}_{\theta}\right)^{2}} \beta_{\mathrm{j}_{\mathrm{b}}}-\frac{\mathrm{c}_{\phi} \mathbf{s}_{\theta}}{\left(\mathrm{c}_{\theta}\right)^{2}} \beta_{\mathrm{k}_{\mathrm{b}}} \\
\mathbf{0}_{5} \\
-\frac{\mathbf{s}_{\phi}}{\mathrm{c}_{\theta}} \\
-\frac{\mathrm{c}_{\phi}}{\mathrm{c}_{\theta}} \\
\mathbf{0}_{2}
\end{array}\right]^{\top}
\end{aligned}
$$

For convenience, we will combine the vectors obtained so far into a single observability matrix, $\mathbf{O}_{\mathrm{M}}$. To facilitate the presentation we will temporarily simplify $\mathbf{O}_{\mathrm{M}}$. We note that for our vehicle $\frac{\mu}{m} \approx 0.28$ and gyroscope biases are on the order of $10^{-3}$. We will assume $\phi$ and $\theta$ are small such that $\cos (\phi) \approx 1$, $\sin (\phi) \approx \phi$, etc. We also assume $u, v$, and $w$ are each at most 0.25 meters per second, and we set them to this value to calculate entries in $\mathbf{O}_{\mathrm{M}}$. We justify the assumptions based on the aforementioned need of the hexacopter's sensors to maintain a modest velocity. 
Using these values and assumptions the observability matrix can be approximated as

$$
\mathbf{O}_{\mathrm{M}} \triangleq\left[\begin{array}{c}
\mathbf{d} h_{1} \\
\mathbf{d} h_{2} \\
\mathbf{d} L_{\mathbf{f}} h_{1} \\
\mathbf{d} L_{\mathbf{f}} h_{2} \\
\mathbf{d} h_{3} \\
\mathbf{d} h_{4} \\
\mathbf{d} h_{5} \\
\mathbf{d} h_{6} \\
\mathbf{d} L_{\mathbf{f}} h_{3} \\
\mathbf{d} L_{\mathbf{f}} h_{4} \\
\mathbf{d} L_{\mathbf{f}} h_{5} \\
\mathbf{d} L_{\mathbf{f}} h_{6}
\end{array}\right] \approx\left[\begin{array}{cccccccccccccc}
0 & 0 & 0 & 0 & 0 & 0 & -\frac{\mu}{m} & 0 & 0 & 0 & 0 & 0 & 1 & 0 \\
0 & 0 & 0 & 0 & 0 & 0 & 0 & -\frac{\mu}{m} & 0 & 0 & 0 & 0 & 0 & 1 \\
0 & 0 & 0 & 0 & \frac{g \mu}{m} & 0 & 0 & 0 & 0 & 0 & 0 & 0 & 0 & 0 \\
0 & 0 & 0 & -\frac{g \mu}{m} & 0 & 0 & 0 & 0 & 0 & 0 & 0 & 0 & 0 & 0 \\
1 & 0 & 0 & 0 & 0 & 0 & 0 & 0 & 0 & 0 & 0 & 0 & 0 & 0 \\
0 & 1 & 0 & 0 & 0 & 0 & 0 & 0 & 0 & 0 & 0 & 0 & 0 & 0 \\
0 & 0 & 1 & 0 & 0 & 0 & 0 & 0 & 0 & 0 & 0 & 0 & 0 & 0 \\
0 & 0 & 0 & 0 & 0 & 1 & 0 & 0 & 0 & 0 & 0 & 0 & 0 & 0 \\
0 & 0 & 0 & w \mathrm{~s}_{\psi} & w \mathrm{c}_{\psi} & o_{1} & \mathrm{c}_{\psi} & -\mathrm{s}_{\psi} & 0 & 0 & 0 & 0 & 0 & 0 \\
0 & 0 & 0 & -w \mathrm{c}_{\psi} & w \mathrm{~s}_{\psi} & o_{2} & \mathrm{~s}_{\psi} & \mathrm{c}_{\psi} & 0 & 0 & 0 & 0 & 0 & 0 \\
0 & 0 & 0 & v & -u & 0 & 0 & 0 & 1 & 0 & 0 & 0 & 0 & 0 \\
0 & 0 & 0 & 0 & 0 & 0 & 0 & 0 & 0 & 0 & 0 & -1 & 0 & 0
\end{array}\right],
$$

where $o_{1} \triangleq-u \mathrm{~s}_{\psi}-v \mathrm{c}_{\psi}$ and $o_{2} \triangleq u \mathrm{c}_{\psi}-v \mathrm{~s}_{\psi}$. We have grouped rows in $\mathbf{O}_{\mathrm{M}}$ according to their corresponding sensor type. The first four rows derive from accelerometer measurements. The remaining rows depend on the exteroceptive sensor algorithm. The most significant entries in $\mathbf{O}_{\mathrm{M}}$ are between approximately 0.5 and 3.0 in magnitude.

The columns lacking significant entries in (26) correspond to the states $\beta_{\mathrm{i}_{\mathrm{b}}}$ and $\beta_{\mathrm{j}_{\mathrm{b}}}$. This leads us to consider other candidate vectors for $\mathbf{O}_{\mathrm{M}}$ that would have significant entries in these positions. Vectors of the form $\mathbf{d} L_{\mathbf{g}_{j}} h_{n}$ have only zero in these columns. We therefore consider second order derivative vectors of the form $\mathbf{d} L_{\mathbf{g}_{j}} L_{\mathbf{f}} h_{n}$ or $\mathbf{d} L_{\mathbf{f}} L_{\mathbf{f}} h_{n}$.

Again using the values and approximations mentioned above, the only likely candidates are $\mathbf{d} L_{\mathbf{f}} L_{\mathbf{f}} h_{1}$ and $\mathbf{d} L_{\mathbf{f}} L_{\mathbf{f}} h_{2}$. These vectors can be simplified as

$$
\mathbf{d} L_{\mathbf{f}} L_{\mathbf{f}} h_{1} \approx\left[\begin{array}{c}
0 \\
0 \\
0 \\
0 \\
0 \\
0 \\
0 \\
0 \\
0 \\
0 \\
-\frac{g \mu}{m} \\
0 \\
0 \\
0
\end{array}\right]^{\top}, \quad \mathbf{d} L_{\mathbf{f}} L_{\mathbf{f}} h_{2} \approx\left[\begin{array}{c}
0 \\
0 \\
0 \\
0 \\
0 \\
0 \\
0 \\
0 \\
0 \\
\frac{g \mu}{m} \\
0 \\
0 \\
0 \\
0
\end{array}\right]^{\top}
$$

The term $\frac{g \mu}{m} \approx 2.8$ provides the necessary entries in the $10^{\text {th }}$ and $11^{\text {th }}$ columns. When we augment $\mathbf{O}_{\mathrm{M}}$ with these two rows, the approximate representations in (26) and (27) yield a full rank, well conditioned matrix. To facilitate further 
discussion we will indicate a single element of the matrix with the notation $\mathbf{O}_{\mathrm{M}}($ row, column $)$.

We can see that the improved model for accelerometer measurements leads to significant entries at $\mathbf{O}_{\mathrm{M}}(1,7)$ and $\mathbf{O}_{\mathrm{M}}(2,8)$. These columns correspond to $u$ and $v$, the two components of velocity in the plane of the rotors. Without these entries arising from the improved model, observing $u$ and $v$ would totally rely upon the position measurements $h_{3}$ and $h_{4}$, as indicated by the nonzero elements at $\mathbf{O}_{\mathrm{M}}(9,7), \mathbf{O}_{\mathrm{M}}(9,8), \mathbf{O}_{\mathrm{M}}(10,7)$, and $\mathbf{O}_{\mathrm{M}}(10,8)$.

We also note that the improved model for accelerometer measurements leads to significant values at $\mathbf{O}_{M}(3,5)$ and $\mathbf{O}_{M}(4,4)$. These columns correspond to $\phi$ and $\theta$. Having significant entries at $\mathbf{O}_{\mathrm{M}}(3,5)$ and $\mathbf{O}_{\mathrm{M}}(4,4)$ supports our assertion at the end of Section 2.3. The improved dynamic model ought to improve attitude estimates because deflections from hover cause the accelerations and therefore the velocities that the accelerometers measure.

In the preceding development we have highlighted the important structure of $\mathbf{O}_{\mathrm{M}}$ by omitting less significant terms in (26) and (27). To further verify observability we evaluate the actual expressions in $\mathbf{O}_{\mathrm{M}}$ over time for an entire flight. We use a motion capture system to measure the vehicle's position and orientation during the flight. We also use this high rate $(\approx 200 \mathrm{~Hz})$ information to estimate the vehicle's velocity in the body-fixed reference frame. The only

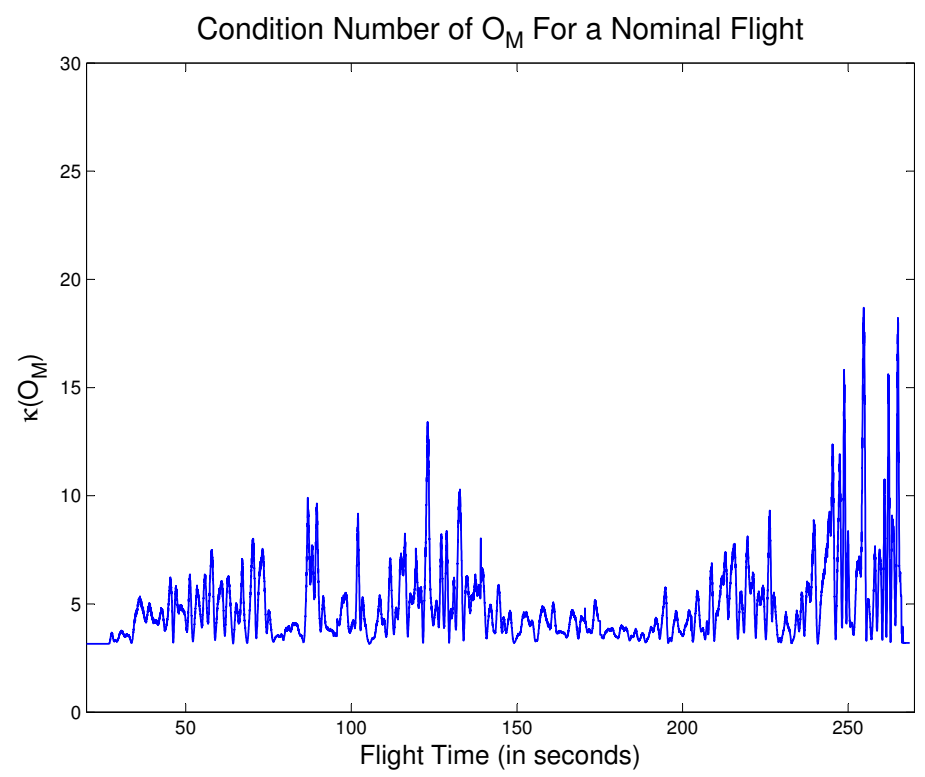

Fig. 3 This figure shows the condition number of the observability matrix as a function of time for the nominal flight used in Section 4. The condition number, $\kappa\left(\mathbf{O}_{M}\right)$, is defined as the ratio of the largest to the smallest singular value of $\mathbf{O}_{\mathrm{M}}$. This figure represents the condition number for $\mathbf{O}_{\mathrm{M}}$ without approximations other than neglecting terms multiplied by gyroscope biases. 
approximation we retain is to omit terms multiplied by gyroscope biases. The biases are distinctly small in magnitude, and we have no way to accurately determine their true value. In Figure 3 we plot the condition number of $\mathbf{O}_{\mathrm{M}}$ over a typical flight and note that $\mathbf{O}_{\mathrm{M}}$ is full rank for the duration.

\section{Results}

\subsection{Experimental Setup}

Our purpose in this paper is to study the characteristics of our state estimation approach relative to approaches common in the literature. We collect ground truth data using a motion capture system. ${ }^{3}$ The following results were generated using a commercial hexacopter available from MikroKopter. ${ }^{4}$ The IMU included with the vehicle outputs data at approximately $40 \mathrm{~Hz}$. We do not use actual sensors and algorithms to generate measurements of position and heading; we instead synthesize these with downsampled and manipulated data from our motion capture system.

Synthesizing measurement data for Equations (20) - (23) allows us to easily adjust the update rate and noise characteristics. Downsampled data provides synthesized measurements at any rate from $2-30 \mathrm{~Hz}$. We also randomly delay the measurements with a mean delay of $250 \mathrm{~ms}$ to simulate the time needed to process exteroceptive sensor data. Finally, we add various levels of independent Gaussian noise to each of the synthesized measurements as described in the results below. While there are certainly artifacts of real sensor processing not captured by this approach, we assume it is sufficient for a comparative analysis.

For comparison we have implemented a scheme common among researchers using multirotor helicopters. In this traditional approach a low-level filter estimates roll and pitch angles, $\phi_{\mathrm{I}}$ and $\theta_{\mathrm{I}}$, based only on gyroscope and accelerometer data. These attitude estimates are based on the typical but faulty assumption that the accelerometers onboard the hexacopter can measure the gravity vector $[12]$.

The estimates of $\phi_{\mathrm{I}}$ and $\theta_{\mathrm{I}}$ from the low-level filter are estimated independent of the remaining states; they are passed along with gyro and accelerometer data as inputs into a separate CD-EKF. The states estimated by this filter include position, heading, and velocity. Position and heading are propagated in accordance with Equations (6) and (7), but velocity states propagate using the model

$$
\left[\begin{array}{c}
\dot{u} \\
\dot{v} \\
\dot{w}
\end{array}\right]=\mathbf{a}_{\mathrm{m}}^{\mathrm{b}}+\mathbf{R}_{\mathrm{L}}^{\mathrm{b}}\left(\phi_{\mathrm{I}}, \theta_{\mathrm{I}}\right) \mathbf{g}
$$

where $\mathbf{a}_{\mathrm{m}}^{\mathrm{b}}$ represents the three axis accelerometer measurements in the body frame, $\mathbf{R}_{\mathrm{L}}^{\mathrm{b}}\left(\phi_{\mathrm{I}}, \theta_{\mathrm{I}}\right)$ is the rotation matrix from the local to the body frame

\footnotetext{
3 http://www.motionanalysis.com/

4 http://www.mikrokopter.de
} 
based on $\phi_{\mathrm{I}}$ and $\theta_{\mathrm{I}}$, and $\mathbf{g}$ is the gravity vector expressed in the local reference frame. The position and heading measurements provide the only correction in this traditional approach.

Equation (28) is a valid method for propagating velocity estimates only if $\mathbf{R}_{\mathrm{L}}^{\mathrm{b}}$ is correct. The false assumption used to generate $\phi_{\mathrm{I}}$ and $\theta_{\mathrm{I}}$ makes these estimates modestly but consistently inaccurate. We have taken care to make our estimates of $\phi_{\mathrm{I}}$ and $\theta_{\mathrm{I}}$ qualitatively match those from a popular commercial multirotor platform.

To be concise in presenting results we do not present below the estimation results for $d, w$, or $\psi$. The improved dynamic model we have presented does not offer any direct benefit in these states over the traditional approach. We will also omit discussing the estimation results for gyroscope biases. These biases can be easily recalibrated just before flight and do not evolve significantly over short flights like the one presented below. However, including gyroscope biases in the state would be especially significant in scenarios where the vehicle flies autonomously for longer periods, such as when it can land and recharge autonomously [16].

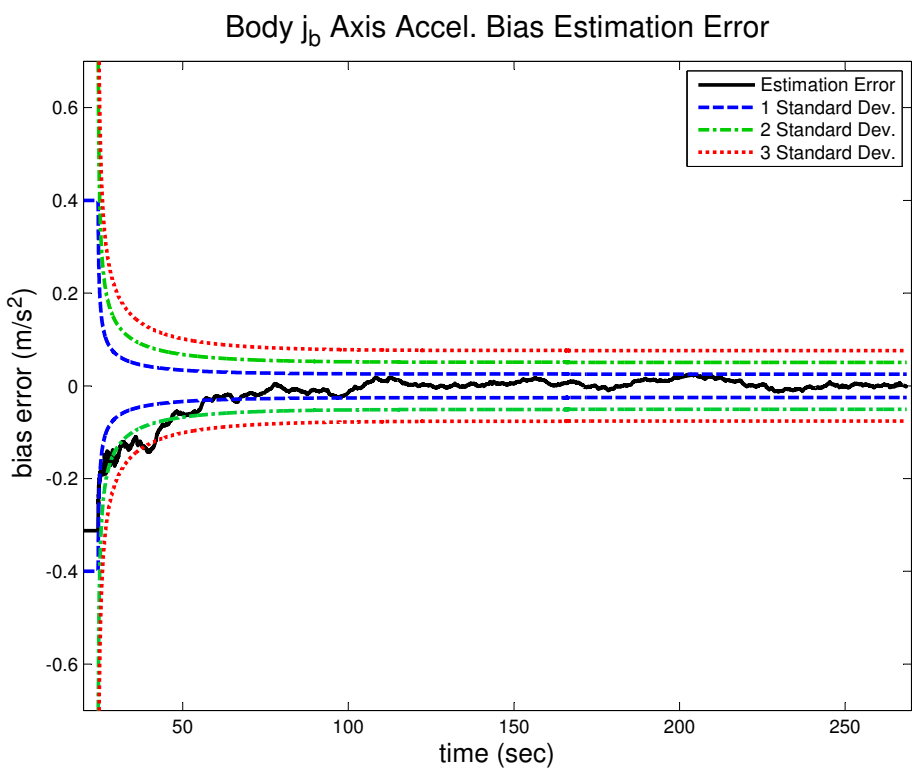

Fig. 4 Error of the accelerometer bias $\alpha_{\mathrm{j}_{\mathrm{b}}}$ estimate over the entire flight when initialized to zero (i.e. uncalibrated). Results for $\alpha_{\mathrm{i}_{\mathrm{b}}}$ are similar. The error is plotted as the solid black line. The remaining curves designate multiples of the marginal standard deviation of the error as calculated by the filter.

The yaw angle $\psi$ is kept close to zero for the majority of this four minute flight. When $\psi=0$, then $f_{\mathrm{L}}, \theta$, and $u$ are decoupled from $r_{\mathrm{L}}, \phi$, and $v$ (i.e. changes in $v$ caused by $\phi$ are solely responsible for changes in $r_{\mathrm{L}}$ ). We will 
omit plots of $f_{\mathrm{L}}, \theta$, and $u$ since they are qualitatively similar to the plots of $r_{\mathrm{L}}, \phi$, and $v$.

Finally, we make a brief observation about accelerometer biases. Typical hardware implementations can require a careful calibration of accelerometer biases before flight. We performed such a calibration and used the calibrated bias values in the results for the traditional approach. However, we chose to disadvantage the filter using the improved model by initializing its accelerometer biases to zero. Figure 4 shows that the filter estimated value for these biases quickly converges, and the results for the other state estimates suggest this lack of prior calibration is not a problem for the filter using the improved model.

\subsection{State Estimation Results}

Figures $5-7$ show results for the first minute of a manually controlled, nominal flight. We note in Figure 5 that errors in $\phi_{\mathrm{I}}$ used in (28) do not appear exceptionally large. The most noticeable discrepancies are on the order of 10 degrees. The traditional approach also leads to errors for translational displacement in Figure 6 that seem somewhat tolerable. Although the displacement errors shown here can be as much as 0.5 meters, they are brief and only at their worst after a rapid transition.

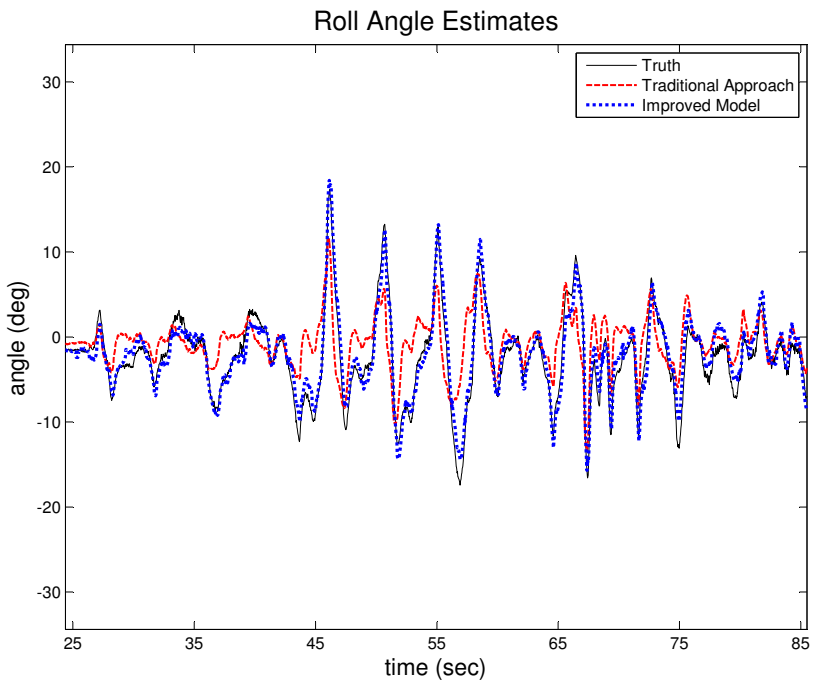

Fig. 5 Estimates of roll angle, $\phi$, during the first minute of a manually controlled flight. These results were generated using exteroceptive position updates arriving at $5 \mathrm{~Hz}$, delayed on average by $250 \mathrm{~ms}$, and with $5 \mathrm{~cm}$ standard deviation of error. Flight begins at about $t=25$ seconds. 


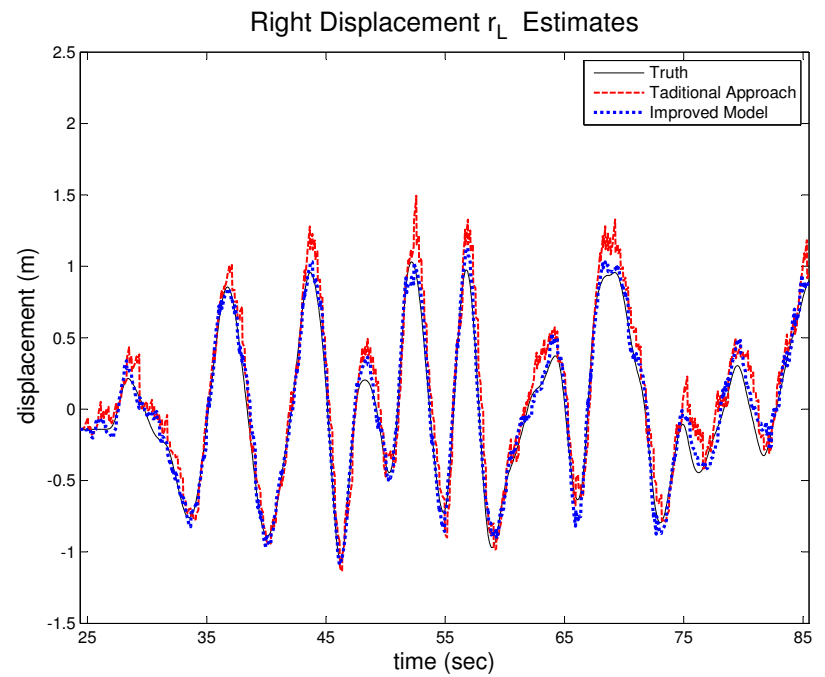

Fig. 6 Estimates of right displacement from the local reference frame, $r_{\mathrm{L}}$. See also the caption on Figure 5

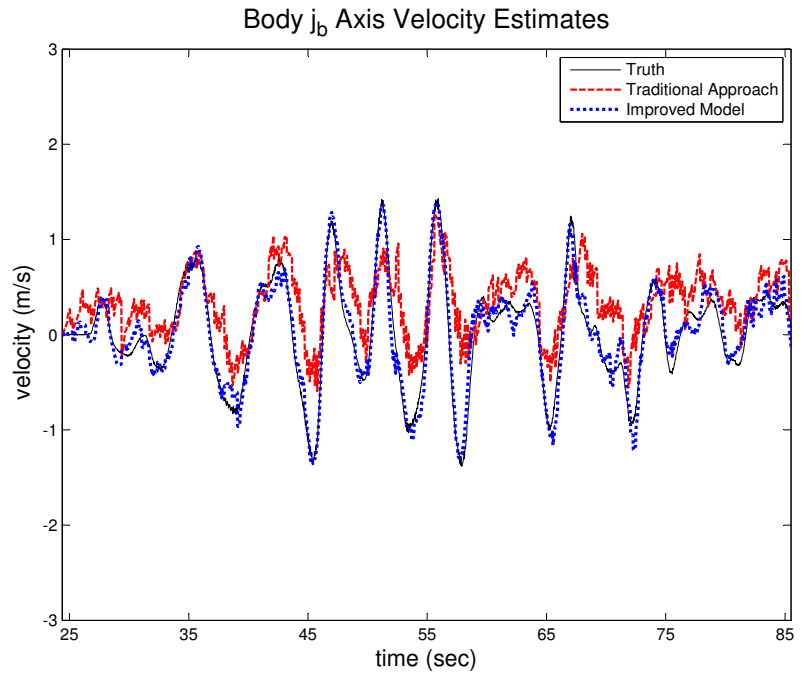

Fig. 7 Estimates of the body y-axis velocity, $v$. See also the caption on Figure 5

However, the significance of errors in $\phi_{\mathrm{I}}$ becomes more apparent when estimating the corresponding component of velocity. This is illustrated in Figure 7. The relatively infrequent rate $(5 \mathrm{~Hz})$ of position updates in these results cannot adequately compensate for the errors introduced in (28) by the incorrect 
rotation matrix. While the traditional estimate of $v$ trends correctly, it can be off by as much as $1.5 \mathrm{~m} / \mathrm{s}$. This is despite relatively accurate exteroceptive updates.

The hexacopter depends on accurate state estimates, especially estimates of velocity, to control its fast dynamics. Feeding back poor velocity estimates into a controller would have a deleterious effect on flight performance. However, estimates of velocity found by using the improved model closely track the truth.

The more accurate estimates afforded by the improved model are also robust to decreased accuracy in the exteroceptive measurements. This is quantified in Figure 8 where we plot the RMS error over the entire four minute flight for the roll, velocity, and position displacement. The RMS errors for our observer and the traditional approach using (28) are calculated for three different levels of error in the exteroceptive sensor algorithm's measurement of position and heading.
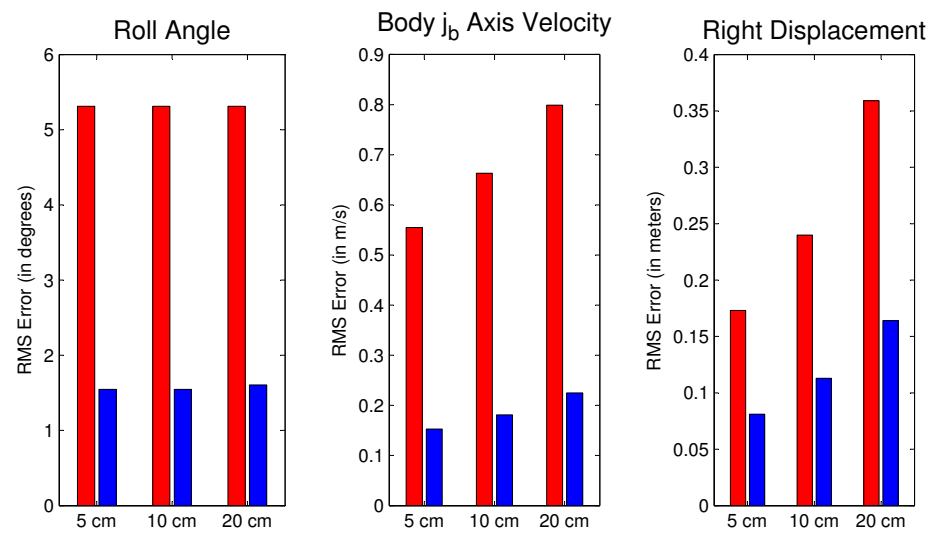

Fig. 8 Average error in roll angles $\phi$ and $\phi_{\mathrm{I}}$ (left), body y-axis velocity $v$ (center), and relative right displacement $r_{\mathrm{L}}$ (right) over a four minute flight. Error of the traditional approach using (28) is graphed in red (light gray); error of estimates based on the improved model are graphed in blue (dark gray). Error is calculated for three scenarios differing in the level noise in the exteroceptive position update. Position measurements are provided at $5 \mathrm{~Hz}$.

We note in Figure 8 that the estimates of $\phi_{\mathrm{I}}$ are unaffected by changes in exteroceptive errors. This should be expected because $\phi_{\mathrm{I}}$ is estimated in a separate observer. However, it is worth noting that estimates of $\phi$ generated by the filter using the improved model are also essentially unchanged by the quality of exteroceptive updates. The first four rows of $\mathbf{O}_{\mathrm{M}}$ in (26) suggest that the roll and pitch angles are observable based only on accelerometer measurements. That property is evident by the robustness shown here.

As expected, Figure 8 also demonstrates a more graceful degradation in position and velocity estimates when using the improved model. Position and 
velocity states suffer more under the traditional approach relying on (28) because there is no direct correction of velocity. As noted earlier by [18], high rate position information is essential in the traditional approach.

\subsection{Varying the Exteroceptive Update Rate}

The foregoing results highlight the complementary relationship between the improved dynamic model and the position corrections from exteroceptive sensor processing. The improved model allows velocity estimates to be corrected directly by relatively frequent accelerometer data instead of relying heavily on measurements of position. Better velocity estimates in turn improve position estimates through their kinematic relationship making all of the estimates more robust as the position measurements become less accurate.

In this subsection we further test the benefit of that relationship by varying the rate at which we apply the exteroceptive measurements of position and heading. The position measurements in these results are delayed on average by 0.2 seconds and corrupted by zero mean Gaussian noise with a standard deviation of 0.1 meters. Update rates range from 2 to $30 \mathrm{~Hz}$. In the legends we designate the traditional approach EKF-t while the filter using the improved model is referred to simply as EKF.

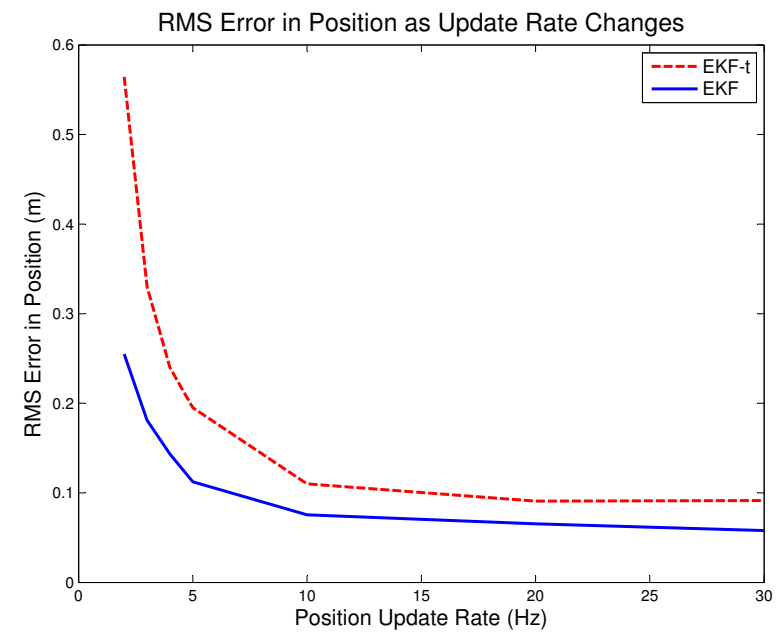

Fig. 9 RMS error in position with respect to position update rate.

Figure 9 demonstrates the RMS error in position estimates. For any given update rate the position estimates of the EKF are about twice as accurate as those from EKF-t. Examining horizontal lines through the plot shows the position update rate for the EKF can be cut about in half while maintaining the same degree of accuracy as EKF-t. 


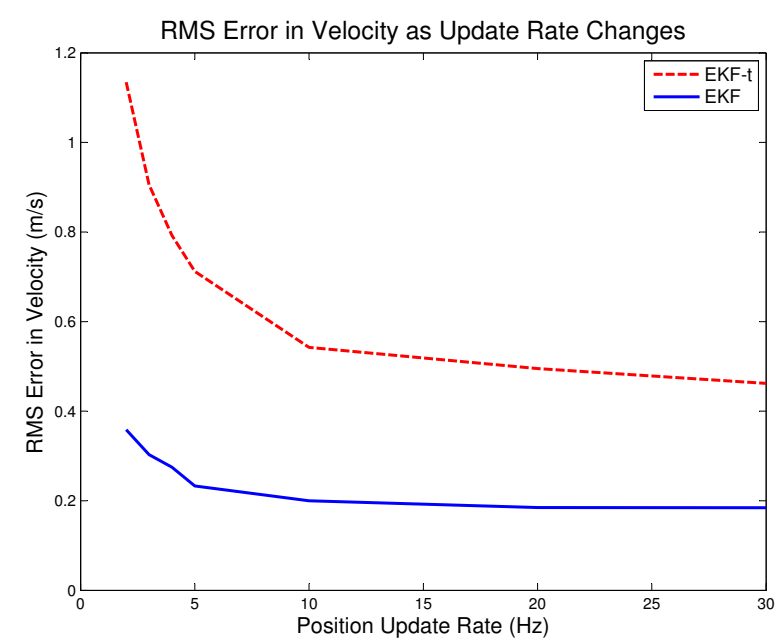

Fig. 10 RMS error in velocity with respect to position update rate.

Figure 10 demonstrates the RMS error in velocity estimates. The EKF outperforms EKF-t by at least a factor of two even when position measurements are available at $30 \mathrm{~Hz}$, the framerate of many digital cameras. As the rate of position measurements decreases the gap between the two filters is even more pronounced. The reduced demand on algorithms providing position measurements is especially significant when those measurements must be generated onboard the helicopter's limited computer and without prior knowledge of the environment.

\section{Conclusions and Future Work}

We have presented and analyzed an observer based on an improved dynamic model for multirotor helicopters. The filter produces more accurate attitude and velocity estimates compared to traditional models based on (28) because it correctly accounts for the relationship between accelerometer measurements and velocity in the plane of the rotors. We have motivated the improved estimation by analyzing the observability properties of the filter. The increased accuracy provided by this observer relaxes constraints on developing an appropriate exteroceptive sensor-based position update. Using the model presented here, both accelerometer and exteroceptive measurements can be used to more effectively update the velocity estimates so critical to autonomous control of these vehicles.

The results presented here could be further improved by more frequent access to gyroscope and accelerometer measurements. Using IMU data at $40 \mathrm{~Hz}$ is modest compared to rates commonly reported in the literature (e.g. [5]). We used this rate only because of the current hardware limitations of our prototype testbed. However, improvements due to faster access to the IMU may not be 
worth the increased computation given the current quality of the estimates presented here.

Future work will center on integrating this observer with a vision-based position estimation algorithm similar to [7]. Their algorithm develops a globally consistent SLAM map that would enable the autonomous vehicle to explore completely unknown terrain. Outputs from that vision algorithm would be used as inputs to the filter described in the work.

\section{Acknowledgements}

This work was supported by the DoD SMART Scholarship program.

\section{References}

1. Bachrach, A., Prentice, S., He, R., Roy, N.: RANGE - Robust Autonomous Navigation in GPS-denied Environments. Journal of Field Robotics 28(5), 644-666 (2011)

2. Bills, C., Chen, J., Saxena, A.: Autonomous MAV Flight in Indoor Environments Using Single Image Perspective Cues. In: IEEE Int. Conf. on Robotics and Automation, pp. 5776-5783 (2011)

3. Bouabdallah, S.: Design and Control of Quadrotors with Application to Autonomous Flying. Phd, ÉCOLE POLYTECHNIQUE FÉDÉRALE DE LAUSANNE (2007)

4. Bresciani, T.: Modelling, Identification and Control of a Quadrotor Helicopter. Master thesis, Lund University (2008)

5. Gurdan, D., Stumpf, J., Achtelik, M., Doth, K.M., Hirzinger, G., Rus, D.: Energyefficient Autonomous Four-rotor Flying Robot Controlled at $1 \mathrm{kHz}$. In: IEEE Int. Conf. on Robotics and Automation, April, pp. 10-14 (2007)

6. Hehn, M., Andrea, R.D.: Quadrocopter Trajectory Generation and Control. In: International Federation of Automatic Control (IFAC) World Congress (2011)

7. Konolige, K., Bowman, J., Chen, J.D., Mihelich, P., Calonder, M., Lepetit, V., Fua, P.: View-based Maps. The International Journal of Robotics Research 29(8), 941-957 (2010)

8. Kumar, V., Michael, N.: Opportunities and Challenges with Autonomous Micro Aerial Vehicles. In: 15th International Sympossium on Robotics Research (2011)

9. Macdonald, J.C.: Efficient Estimation for Autonomous Multi-Rotor Helicopters Operating in Unknown, Indoor Environments. Ph.d., Brigham Young University (2012)

10. Madani, T., Benallegue, A.: Backstepping Control for a Quadrotor Helicopter. 2006 IEEE/RSJ International Conference on Intelligent Robots and Systems pp. 3255-3260 (2006)

11. Madani, T., Benallegue, A.: Control of a Quadrotor Mini-Helicopter via Full State Backstepping Technique. Proceedings of the 45th IEEE Conference on Decision and Control pp. 1515-1520 (2006)

12. Martin, P., Salaün, E.: The True Role of Accelerometer Feedback in Quadrotor Control. IEEE International Conference on Robotics and Automation pp. 1623-1629 (2010)

13. Meier, L., Tanskanen, P., Fraundorfer, F., Pollefeys, M.: PIXHAWK : A System for Autonomous Flight using Onboard Computer Vision. In: IEEE Int. Conf. on Robotics and Automation, pp. 2992-2997 (2011)

14. Mellinger, D., Kumar, V.: Minimum Snap Trajectory Generation and Control for Quadrotors. In: IEEE International Conference on Robotics and Automation, pp. 2520$2525(2011)$

15. Michini, B., Redding, J., Ure, N.K., Cutler, M., How, J.P.: Design and Flight Testing of an Autonomous Variable-Pitch Quadrotor. In: IEEE International Conference on Robotics and Automation, pp. 2978-2979 (2011) 
16. Redding, J., Dydek, Z., How, J.P., Vavrina, M.A., Vian, J.: Proactive Planning for Persistent Missions using Composite Model-Reference Adaptive Control and Approximate Dynamic Programming. In: American Control Conference, pp. 2332-2337 (2011)

17. Rondon, E., Garcia-Carrillo, L., Fantoni, I.: Vision-Based Altitude, Position and Speed Regulation of a Quadrotor Rotorcraft. In: Intelligent Robots and Systems (IROS), 2010 IEEE/RSJ International Conference on, pp. 628-633 (2010)

18. Sa, I., Corke, P.: System Identification, Estimation and Control for a Cost Effective Open-Source Quadcopter. In: IEEE Int. Conf. on Robotics and Automation, pp. 22022209 (2012)

19. Shen, S., Michael, N., Kumar, V.: Autonomous Multi-Floor Indoor Navigation with a Computationally Constrained MAV. In: IEEE International Conference on Robotics and Automation, pp. 20-25 (2011)

20. Thrun, S., Leonard, J.J.: Simultaneous Localization and Mapping. In: B. Siliciano, O. Khatib (eds.) Handbook of Robotics, chap. 37, pp. 871-889. Springer (2008)

21. Vidyasagar, M.: Nonlinear systems analysis (2nd ed.). Prentice-Hall, Inc., Upper Saddle River, NJ, USA (1992)

22. Xu, R., Ozguner, U.: Sliding Mode Control of a Quadrotor Helicopter. Proceedings of the 45th IEEE Conference on Decision and Control pp. 4957-4962 (2006) 